\title{
Transforming Public Health Surveillance Through Open Public Health Information
}

\author{
Janelle Kibler ${ }^{2}$, Dr. Scott McNabb*1, James Lavery ${ }^{1}$, Ziad Memish ${ }^{3}$, Affan Shaikh ${ }^{4}$ and \\ Ngozi Erondu ${ }^{5}$
}

${ }^{1}$ Rollins School of Public Health, Emory University, Atlanta, GA, USA; ${ }^{2}$ Emory University, Atlanta, GA, USA; ${ }^{3}$ WHO, Riyadh, Saudi Arabia; ${ }^{4}$ Public Health Practice, Grass Valley, CA, USA; ${ }^{5}$ London School of Hygiene and Tropical Medicine, London, United Kingdom

\begin{abstract}
Objective
The goal of this editorial is to shed light on the lack of transparency that exists in the sharing of Public Health data and to reverse this presumption in favour of open public health information properly vetted and openly accessible. Open public health information is a critical step to revitalize public health practice and is a human right.
\end{abstract}

\section{Introduction}

Public health practice that prevents, detects, and responds to communicable and noncommunicable disease threats is hindered by poor access to public health data and information. This includes timely sharing of case-based information, respecting patent and publication rights, and the ethical sharing of specimens. Disagreements about information shared and under what circumstances plus who has right to the data, clinical specimens, and their derivative products impede research and countermeasures. Delayed or inaction by public health authorities undermines trust and exacerbates the crisis. Evident in 2014 by the delayed Public Health Emergency of International Concern declaration of the Ebola virus outbreak in West Africa by the World Health Organization, the governing presumption is that access to public health information should be restricted, constrained, or even hoarded; this is a failed approach. This lack of transparency prevents information availability when and where it is needed and obstructs public health efforts to efficiently and ethically prevent, detect, and respond to emerging threats. A better way forward is to reverse this presumption in favour of open public health information properly vetted and openly accessible. Open public health information is a critical step to revitalize public health practice and is a human right.

While there is limited global consensus among scientists and public health practitioners on best practices to guide national health authorities, researchers, NGOs, and industry as they navigate the ethical, political, technical, and economic challenges associated with the sharing of essential public health information (e.g., pathogen isolates, clinical specimens, and patient-related data), grounding this discussion on the guiding principles of open public health information can help navigate the complex privacy, security, communication, and access needs, and ensure that collaboration and sharing occur in a manner that is ethically and socially just, efficient, and equitable. Built on existing governance frameworks such as the International Health Regulations (IHRs) and the Pandemic Influenza Preparedness Framework (PIP), open public health can transform public health surveillance, allowing for the rapid sharing of data and products during outbreaks for mutual benefit and enhanced global health security.

\section{Methods}

This abstract represents a larger editorial style manuscript, thus no methods were developed in the abstract.

\section{Results}

This editorial style manuscript aims to reverse the presumption that public health data is damaging to one in favour of open public health information properly vetted and openly accessible.

\section{Conclusions}

Similar to other open movements (i.e., open data, open government, open development, and open science) that seek to address the world's greatest challenges through transparency, collaboration, reuse of and free access to ideas, open public health offers an ideal solution to overcome the challenges in the 21 st century.

\section{Keywords}

public health data; data transparency; open data

\section{*Dr. Scott McNabb}

E-mail: scottjnmcnabb@emory.edu 